OPEN ACCESS

Edited by:

Isabelle Cremer,

Sorbonne Universités, France

Reviewed by:

Alessandro Rizzo,

Sant'Orsola-Malpighi Polyclinic, Italy

Antonio Facciorusso,

University of Foggia, Italy

*Correspondence:

Ruili Zhao

ebhzhaoruili@126.com

Zhanjun Guo

zjguo5886@aliyun.com

Specialty section:

This article was submitted to

Cancer Immunity

and Immunotherapy,

a section of the journal

Frontiers in Immunology

Received: 13 October 2021 Accepted: 17 November 2021 Published: 07 December 2021

Citation:

Xu S, Lai R, Zhao $Q$, Zhao $P$, Zhao $R$ and Guo $Z$ (2021) Correlation Between Immune-Related Adverse Events and

Prognosis in Hepatocellular

Carcinoma Patients Treated With Immune Checkpoint Inhibitors.

Front. Immunol. 12:794099. doi: 10.3389/fimmu.2021.794099

\section{Correlation Between Immune- Related Adverse Events and Prognosis in Hepatocellular Carcinoma Patients Treated With Immune Checkpoint Inhibitors}

\author{
Shuo Xu ${ }^{1}$, Ruixue Lai ${ }^{1}$, Qian Zhao ${ }^{1}$, Pandong Zhao ${ }^{1}$, Ruili Zhao ${ }^{2 *}$ and Zhanjun Guo ${ }^{1 *}$ \\ ${ }^{1}$ Department of Rheumatology and Immunology, The Fourth Hospital of Hebei Medical University, Shijiazhuang, China, \\ 2 Department of Otolaryngology, The Fourth Hospital of Hebei Medical University, Shijiazhuang, China
}

Background: Immune-related adverse events (irAEs) caused by immune checkpoint inhibitors (ICls) were associated with clinical benefit in cancer patients of melanoma, a lung cancer. In the present study, we investigated the correlation between irAE and ICl efficacy in hepatocellular carcinoma (HCC) patients.

Methods: We divided the HCC patients who received the anti-PD-1 antibody into two groups as irAE group and non-irAE group according to the National Cancer Institute Common Terminology Criteria for Adverse Events ver. 4.03. The treatment efficacy of $\mathrm{ICls}$ was evaluated with objective response rate (ORR), disease control rate (DCR), progression-free survival (PFS), and overall survival (OS).

Result: Of the 65 HCC patients who received the anti-PD-1 antibody (monotherapy or combined with targeted medicine), median PFS in the irAE group was superior to that in the non-irAE group (302 days vs. 148 days, $p=0.004$ ). Median OS in the irAE group was also better than that in the non-irAE group (374 days vs. 279 days, $p=0.038$ ). Although the statistical difference for DCR in the irAE group and non-irAE group was not reached, the DCR of the irAE displayed a trend better than that of the non-irAE group $(41.20 \%$ vs. $20.80 \%, p=0.118$ ). Multivariate analysis also demonstrated that the non-irAE group $(\mathrm{HR}=6.410,95 \% \mathrm{Cl}: 1.404$ to 29.275$)$ was associated independently with the poor prognosis.

Conclusions: Development of irAEs was associated with clinical benefit for HCC patients who were treated with immune checkpoint inhibitors; irAE, particularly low-grade irAE, was a predictable marker for better $\mathrm{ICl}$ treatment efficiency in $\mathrm{HCC}$ patients.

Keywords: hepatocellular carcinoma, immune-related adverse events, immune checkpoint inhibitors, anti-PD-1 antibody, OS, PFS 


\section{INTRODUCTION}

Hepatocellular carcinoma (HCC) is an important global health issue (1), with approximately 906,000 new cases and 830,000 deaths, making it the sixth most commonly cancer and the third leading cause of cancer death (2). The first-line treatment for unresectable HCC included multi-targeted tyrosine kinase inhibitors (TKI) sorafenib and lenvatinib (3-5), the second-line therapies including regorafenib, cabozantinib, and ramucirumab (6-8), but these drugs prolonged the HCC lifespan with a limited increase.

Immune checkpoint inhibitors (ICIs) have recently been a source of promising new cancer treatments that increase the cytotoxicity of $\mathrm{T}$ cells by blocking intrinsic downregulators of immunity such as cytotoxic T-lymphocyte antigen 4 (CTLA-4) and programmed cell death 1 (PD-1) or its ligand of programmed cell death ligand 1 (PD-L1) so as to enhance antitumor activity (9). Recently, ICIs have made a breakthrough in a range of tumor therapies such as advanced non-small-cell lung cancer (NSCLC) (10), melanoma (11), renal cell carcinoma (12), and squamous cell carcinoma of head and neck (SCCHN) (13). In the early phase I/II or phase II studies, the objective response rate (ORR) for advanced HCC people who were treated with the anti-PD-1 antibody of nivolumab or pembrolizumab has been shown to be $14 \%-20 \%$ with prolonged PFS and OS (14-16). However, in the further phase III trials, no satisfying results were obtained for these two antibodies $(17,18)$. The IMbrave150 trial combining the antiPD-L1 antibody of atezolizumab and the anti-VEGF antibody of bevacizumab obtained a breakthrough for advanced HCC treatment referring to ORR, PFS, and OS when compared with sorafenib as the first-line treatment (19-21). In addition, the combination of lenvatinib plus pembrolizumab as a front-line treatment in advanced HCC patients is being evaluated based on its good treatment efficiency in a Phase I study $(22,23)$. In spite of the breakthrough for ICIs on advanced HCC treatment, only a few biomedical predictors of response to ICIs such as microsatellite instability (MSI) and gut microbiome were identified $(24,25)$.

Immune checkpoint inhibitors cause imbalances in immunological tolerance, resulting in inflammatory side effects which are called immune-related adverse events (irAEs) (26). It needs frequent monitoring and immunosuppressive and endocrine therapy based on the severity of the respective $\mathrm{AE}$

\footnotetext{
Abbreviations: ICIs, immune checkpoint inhibitors; irAE, immune-related adverse event; SD, stable disease; $\mathrm{PR}$, partial response; $\mathrm{PD}$, progressive disease; $\mathrm{CR}$, complete response; $\mathrm{ORR}$, objective response rate; $\mathrm{DCR}$, disease control rate; PFS, progression-free survival; OS, overall survival; HCC, hepatocellular carcinoma; TKI, tyrosine kinase inhibitor; PD-1, programmed cell death 1; CTLA-4, cytotoxic T-lymphocyte antigen 4; NSCLC, non-small-cell lung cancer; SCCHN, squamous cell carcinoma of head and neck; ECOG PS, Eastern Cooperative Oncology Group performance status; AFP, alpha fetoprotein; PVTT, portal vein tumor thrombus; TACE, transarterial chemoembolization; HBV, hepatitis B virus; PT, prothrombin time; ALT, alanine aminotransferase; AST, aspartate aminotransferase; NLR, neutrophil-to-lymphocyte ratio; CT, computed tomography; MRI, magnetic resonance imaging; IQR, interquartile range; ECG, electrocardiogram; TMB, tumor mutation burden; MSI-H, microsatellite instability-high.
}

(11, 27-29). The immune-related adverse events are believed to be related to the role that immune checkpoints play in maintaining immunologic homeostasis. The occurrence of irAEs may be related to the following potential mechanisms: increasing T-cell activity against antigens that are present in tumors and healthy tissue, increasing levels of preexisting autoantibodies, an increase in the level of inflammatory cytokines (such as interleukin-17), and enhanced complementmediated inflammation due to direct binding of an antibody against cytotoxic T-lymphocyte antigen 4 (CTLA-4) (9). However, the precise pathophysiology underlying irAEs still needs to be further explored. IrAEs affect almost every organ of the body and are most commonly observed in the skin, gastrointestinal tract, lung, and endocrine, musculoskeletal, and other organs (30).

Recently, several studies have shown that irAEs were associated with efficacy of anti-PD-1 antibody treatment in patients with melanoma, non-small cell lung cancer, and gastric cancer (31-33). In contrast, few data are available considering the relationship between irAE and treatment efficacy of immunotherapy for HCC. In the present study, we investigated the correlation between irAEs and ICIs efficacy in HCC patients.

\section{METHODS}

\section{Patients}

HCC patients with disease stage III or IV who were treated with the anti-PD-1 antibody (monotherapy or combined with targeted medicine) between January 2019 and April 2020 at the Fourth Hospital of Hebei Medical University were involved for analysis. Patients who received other previous treatments with immunotherapy were excluded. We reviewed the medical records, and the following characteristics of patients were collected: age, gender, Eastern Cooperative Oncology Group performance status (ECOG PS), treatment line number, TNM, alpha fetoprotein (AFP), portal vein tumor thrombus (PVTT), number of tumors, history of transarterial chemoembolization (TACE) or surgery, concurrent therapy, metastatic status, history of hepatitis B virus (HBV), baseline blood cell count, prothrombin time (PT), max tumor size, alanine aminotransferase (ALT), aspartate aminotransferase (AST), and albumin and total bilirubin before initiating treatment. The neutrophil-to-lymphocyte ratio (NLR) was calculated by dividing the lymphocyte count into neutrophil count. IrAEs were defined as those inflammatory side effects which were caused by imbalances in immunological tolerance because of the immune checkpoint inhibitors. For the irAE assessment, the National Cancer Institute Common Terminology Criteria for Adverse Events ver. 4.03 was used (https://ctep.cancer.gov/protocolDevelopment/ electronic_applications/ctc.htm\#ctc_40). We divided the patients into two groups, irAE group and non-irAE group, based on occurrence of irAEs.

The study protocol was reviewed and approved by the ethics committee of the Fourth Hospital of Hebei Medical University. Due to the retrospective nature of this study, informed consent was not obtained from each patient. 


\section{Treatment and Assessment}

Patients received a standard anti-PD-1 antibody (monotherapy or combine with targeted medicine) every 3 weeks until disease progression, clinical deterioration, unacceptable toxicity, or patient's refusal. Types of immunotherapy drugs received were camrelizumab, sintilimab, pembrolizumab, and toripalimab, whereas the targeted medicines were apatinib, sorafenib, and lenvatinib (Table S1). Objective tumor response was evaluated by computed tomography (CT) or magnetic resonance imaging (MRI) scan repeated every 2 or 3 cycles according to the RECIST version 1.1.

\section{Statistical Analysis}

Differences between the two groups were compared using the Fisher's exact tests for classified variables and Mann-Whitney U tests for continuous variables. The result of continuous variables was held up as median (interquartile range (IQR)). PFS was defined as the time from the beginning of treatment to progression or death from any cause; in addition, the PFS of patients who survived without disease progression at the end of follow-up was defined as the time from the beginning to the ending date of follow-up. OS was measured until death or censored at the latest follow-up for surviving patients. Probabilities of survival were estimated using the KaplanMeier method and compared using the log-rank test. Univariate analysis and multivariate analysis using a Cox proportional hazard regression model were performed to explore prognostic factors for survival. All statistical analyses were performed using SPSS (IBM SPSS 20.0, NY, USA). All p values are two-sided, and $p<0.05$ was considered to indicate a statistically significant difference.

\section{RESULTS}

\section{Patient Characteristics}

Sixty-nine HCC patients treated with the anti-PD-1 antibody (monotherapy or combine with targeted medicine) were identified as the research subjects. Among them, five patients were excluded because of loss to follow-up and 37 (56.9\%) patients died during the follow-up. The clinical characteristics are listed in Table 1. The median OS and median PFS were 279 days (95\% CI, 268 to 291 days) and 180 days (95\% CI, 140 to 220 days) for overall patients. As shown in Table 2, partial response (PR) was achieved in four patients and stable disease (SD) was observed in 13 patients, which resulted in an ORR of 6.2\% (95\% CI: $0.2 \%$ to $12.2 \%$ ) and DCR of $26.2 \%$ (95\% CI: $15.2 \%$ to $37.1 \%$ ).

\section{Comparison Between irAE and Non-irAE Groups}

The patient backgrounds of the irAE and non-irAE groups are summarized in Table 1. No significant differences in clinical profiles, apart from gender, number of tumors, history of previous treatment, and AST were observed between the two groups.
An ORR of $23.5 \%$ (95\% CI: $1 \%$ to $46 \%$ ) and a DCR of $41.20 \%$ (95\% CI: $15.1 \%$ to $67.3 \%$ ) were observed for all 17 irAE patients (4 PR, 3 SD), whereas only 10 SD patients with DCR of $20.80 \%$ (95\% CI: $8.9 \%$ to $32.8 \%$ ) were achieved in the non-irAE group. Although DCR in the irAE group was superior to that in the nonirAE group, it was not statistically significant $(p=0.118>0.05)$.

The Kaplan-Meier curves of PFS and OS in the irAE and the non-irAE groups are shown in Figure 1. The median PFS of 302 days (95\% CI: 201 to 402 days) for the irAE group was significantly extended compared with the median PFS of 148 days (95\% CI: 104 to 191 days) for the non-irAE group $(p=0.004)$ (Figure 1A). In addition, the median OS was 374 days (95\% CI: 291 to 456 days) in the irAE group and 279 days (95\% CI: 205 to 352 days) in the non-irAE group $(p=0.038)$ (Figure 1B). All of these indicated that irAEs associated with prolonged median PFS and median OS for HCC patients with ICIs treatment.

In addition, we divided the patients with irAEs into two groups: irAE-A group (patients with endocrine, dermatological, or gastrointestinal irAEs) and irAE-B group (patients with other irAEs). The median PFS and median OS of the two groups mentioned above and the non-irAE group were compared pairwise. As shown in Table 3, it is obvious that the median PFS and median OS of the people who experienced endocrine, dermatological, or gastrointestinal irAEs had obvious advantages compared with those who experienced no irAEs (median PFS: 302 vs. 148 days, $p=0.002$; median OS: 374 vs. 279 days, $p=0.034)$.

In the univariate analysis for OS using gender (male or female), ECOG score, treatment line number ( $\leq 2$ or $\geq 3$ ), AFP level ( $<400$ or $\geq 400$ ), number of tumors ( $<3$ or $\geq 3$ ), TNM (III or IV), metastatic status (Yes or No), and irAEs status (non-irAE group or irAE group) as covariates in ICI-treated HCC, clinical characteristics including PS $>1, \geq 3$ treatment line, TNM (IV), AFP $\geq 400$, and tumor metastasis were significantly associated with shorter OS at statistical levels. The non-irAE group was associated with shorter OS at borderline statistical levels (HR = 2.295, 95\% CI: 0.996 to 5.288, $p=0.051$ ). Moreover, multivariate analysis demonstrated that non-irAE (HR $=6.410,95 \% \mathrm{CI}: 1.404$ to $29.275, p=0.017$ ) was independently associated with a poor prognosis (Table 4).

\section{Toxicity}

Seventeen of the 65 patients $(26.15 \%)$ experienced irAEs in our study. Details of these irAEs are shown in Tables S2 and S3. The most frequent adverse event was diarrhea/colitis $(n=6)$, and the second is hypothyroidism $(n=4)$. The median time to onset of irAEs was 68 days (range 26-142 days). There was no grade 3 adverse event observed in our research; no people discontinued the anti-PD-1 antibody because of the irAEs.

\section{DISCUSSION}

Our result indicated that irAEs were associated with better treatment efficacy of ICIs in HCC patients referring to median 
TABLE 1 | Characteristics of patients in irAE and non-irAE groups.

\begin{tabular}{|c|c|c|c|c|}
\hline & Total no. (\%) & Non-irAE no. (\%) & irAE no. (\%) & $p$-value \\
\hline Total N & 65 & 48 & 17 & \\
\hline \multicolumn{5}{|l|}{ Gender } \\
\hline Female & $20(30.8)$ & $10(20.8)$ & $10(58.8)$ & 0.006 \\
\hline Male & $45(69.2)$ & 38 (79.2) & $7(41.2)$ & \\
\hline \multicolumn{5}{|l|}{ Age } \\
\hline$<65$ & $51(78.5)$ & 40 (83.3) & $11(64.7)$ & 0.167 \\
\hline$\geq 65$ & $14(21.5)$ & $8(16.7)$ & $6(35.3)$ & \\
\hline \multicolumn{5}{|l|}{ ECOG PS } \\
\hline$\leq 1$ & $44(67.7)$ & $33(68.8)$ & $11(64.7)$ & 0.770 \\
\hline$>1$ & 21 (32.3) & $15(31.2)$ & $6(35.3)$ & \\
\hline \multicolumn{5}{|l|}{ Treatment line } \\
\hline$\leq 2$ & 59 (90.8) & $42(87.5)$ & $17(100.0)$ & 0.327 \\
\hline$\geq 3$ & $6(9.2)$ & $6(12.5)$ & $0(0.0)$ & \\
\hline \multicolumn{5}{|l|}{ TNM } \\
\hline III & 33 (50.8) & $23(47.9)$ & $10(58.8)$ & 0.574 \\
\hline IV & 32 (49.2) & $25(52.1)$ & 7 (41.2) & \\
\hline \multicolumn{5}{|l|}{ AFP } \\
\hline$<400$ & $41(63.1)$ & $30(62.5)$ & $11(64.7)$ & 1.000 \\
\hline$\geq 400$ & $24(36.9)$ & $18(37.5)$ & $6(35.3)$ & \\
\hline \multicolumn{5}{|l|}{ PVTT } \\
\hline No & $18(27.7)$ & $14(29.2)$ & $4(23.5)$ & 0.760 \\
\hline Yes & 47 (72.3) & $34(70.8)$ & $13(76.5)$ & \\
\hline \multicolumn{5}{|l|}{ Tumor number } \\
\hline$<3$ & $41(63.1)$ & $24(50.0)$ & $17(100.0)$ & 0.001 \\
\hline$\geq 3$ & $24(36.9)$ & $24(50.0)$ & $0(0.0)$ & \\
\hline \multicolumn{5}{|l|}{ Concurrent therapy } \\
\hline No & $11(16.9)$ & $8(16.7)$ & $3(17.6)$ & 1.000 \\
\hline Combine targeted drugs & $54(83.1)$ & $40(83.3)$ & $14(82.4)$ & \\
\hline \multicolumn{5}{|l|}{ Prior therapy } \\
\hline No & $25(38.5)$ & $24(50.0)$ & $1(5.9)$ & 0.001 \\
\hline TACE/surgery & $40(61.5)$ & $24(50.0)$ & $16(94.1)$ & \\
\hline \multicolumn{5}{|l|}{ Metastasis } \\
\hline No & $27(41.5)$ & $20(41.7)$ & $7(41.2)$ & 1.000 \\
\hline Yes & $38(58.5)$ & $28(58.3)$ & $10(58.8)$ & \\
\hline \multicolumn{5}{|l|}{ HBV infection } \\
\hline No & $8(12.3)$ & $6(12.5)$ & $2(11.8)$ & 1.000 \\
\hline Yes & $57(87.7)$ & $42(87.5)$ & $15(88.2)$ & \\
\hline \multicolumn{5}{|l|}{ Child-Pugh score } \\
\hline A & $33(50.8)$ & $21(43.8)$ & $12(70.6)$ & 0.214 \\
\hline B & $24(36.9)$ & $20(41.7)$ & $4(23.5)$ & \\
\hline C & $8(12.3)$ & 7 (14.6) & $1(5.9)$ & \\
\hline \multicolumn{5}{|l|}{ NLR } \\
\hline Low $(\leq 4)$ & $26(40.0)$ & 19 (39.6) & $7(41.2)$ & 1.000 \\
\hline High (>4) & 39 (60.0) & $29(60.4)$ & $10(58.8)$ & \\
\hline \multicolumn{5}{|c|}{ Clinical baseline value (median (IQR)) } \\
\hline PIt & $149.00(123.00,166.00)$ & $149.00(134.00,158.00)$ & $98.00(67.00,180.00)$ & 0.081 \\
\hline WBC & $3.80(3.33,5.28)$ & $3.80(3.34,5.04)$ & $3.33(3.05,5.89)$ & 0.293 \\
\hline Neutrophil & $2.92(2.43,3.81)$ & $2.92(2.43,3.68)$ & $2.44(2.04,4.48)$ & 0.653 \\
\hline Lymphocyte & $0.61(0.52,0.65)$ & $0.60(0.52,0.74)$ & $0.61(0.46,0.65)$ & 0.207 \\
\hline NLR & $4.58(3.48,5.62)$ & $4.58(3.20,5.62)$ & $4.16(3.75,9.88)$ & 0.869 \\
\hline Hemoglobin & $129.00(111.80,137.00)$ & $132.40(111.80,137.00)$ & $121.5(108.0,139.50)$ & 0.167 \\
\hline Max. tumor size & $6.00(4.00,9.50)$ & $7.00(5.00,9.00)$ & $4.00(3.00,10.00)$ & 0.090 \\
\hline PT & $12.70(11.90,13.35)$ & $12.60(11.90,12.80)$ & $12.90(11.85,14.45)$ & 0.558 \\
\hline AST & $65.90(43.50,72.20)$ & $65.90(48.50,72.20)$ & $42.20(30.80,59.10)$ & 0.030 \\
\hline ALT & $31.10(23.50,59.20)$ & $36.95(23.15,87.80)$ & $31.10(23.30,48.70)$ & 0.099 \\
\hline Albumin & $37.70(32.95,42.05)$ & $37.10(30.45,42.05)$ & $39.60(34.30,44.80)$ & 0.307 \\
\hline Total bilirubin & $18.90(15.70,44.25)$ & $31.70(15.85,49.40)$ & $17.00(15.70,42.60)$ & 0.145 \\
\hline
\end{tabular}

ECOG PS, Eastern Cooperative Oncology Group performance status; AFP, alpha fetoprotein; PVTT, portal vein tumor thrombus; TACE, transarterial chemoembolization; HBV, hepatitis B virus; PT, prothrombin time; ALT, alanine aminotransferase; AST, aspartate aminotransferase; NLR, neutrophil-to-lymphocyte ratio.

PFS and median OS, which was compatible with treatment efficacy for irAEs in other tumors, such as melanoma, head and neck squamous cell carcinoma, non-small cell lung cancer, renal cell carcinoma, and urothelial carcinoma $(32,34)$.
We could analyze only a limited number of patient samples, but it is speculated that occurrence of irAEs may be associated with survival even after adjusting other prognostic factors in HCC patients treated with the anti-PD-1 antibody. 
TABLE 2 | Response to immunotherapy.

\begin{tabular}{|c|c|c|c|c|}
\hline Response & Total & irAE group & Non-irAE group & $p$-value \\
\hline PD & 48 & 10 & 38 & - \\
\hline SD & 13 & 3 & 10 & - \\
\hline PR & 4 & 4 & 0 & - \\
\hline CR & 0 & 0 & 0 & - \\
\hline ORR & $6.2 \%$ (95\% Cl: $0.2 \%$ to $12.2 \%)$ & 23.5\% (95\% Cl: 1\% to 46\%) & - & - \\
\hline DCR & $26.2 \%$ (95\% Cl: $15.2 \%$ to $37.1 \%)$ & $41.2 \%(95 \% \mathrm{Cl}: 15.1 \%$ to $67.3 \%)$ & $20.80 \%$ (95\% Cl: $8.9 \%$ to $32.8 \%)$ & 0.118 \\
\hline
\end{tabular}

$S D$, stable disease; $P R$, partial response; $P D$, progressive disease; $C R$, complete response; ORR, objective response rate; $D C R$, disease control rate.

Although DCR or ORR had the advantage in the irAE group, it did not show a statistical difference due to the small sample size. In the previous meta-analysis (35), the occurrences of endocrine, dermatological, and gastrointestinal irAEs were significantly associated with a favorable prognosis in patients treated with ICIs, but other irAEs were not. The occurrence of low-grade, not the high-grade, irAEs was significantly associated with a favorable OS in patients receiving ICIs. Therefore, it is considered reasonable to divide the irAE group into irAE-A group (patients with endocrine, dermatological, or gastrointestinal irAEs) and irAE-B group (patients with other irAEs) and compare them with the non-irAE group in pairs for survival analysis. Our analysis showed that the patients with diarrhea/colitis, hyperthyroidism/hypothyroidism, or rash had a better prognosis. The combined immunotherapy would be mainstream for advanced cancer treatment in the future, while bringing considerable benefits; it will also bring more side effects. Comprehensive judgment based on big data analysis combined with patients' genetic background and different irAE types would be the key to individualized precision therapy.

In our research, there were no grade 3 to 5 adverse events related to the anti-PD-1 antibody and no exacerbation of irAEs after detection, which is probably due to our baseline examination including liver and kidney function, pituitary function, thyroid function, cardiac function, and electrocardiogram (ECG), as well as chest CT every 3 weeks so as to treat irAEs at the early stage. Our results implied that irAEs particularly low-grade irAEs were a predictable marker for better ICI treatment efficiency in HCC patients; we might recommend continuing use for ICI therapy in low-grade irAEs patients. We would not be worried about the occurrence of irAEs in the clinical treatment but exert our utmost effort to prevent fatal irAEs such as immune-associated myocarditis and adrenocortical dysfunction. We would not resist the low-grade irAEs because they provide clinical benefit with low clinical risk.

The occurrence of irAEs during immunotherapy may suggest a higher response rate, but the efficacy of ICIs remains to be limited with an ORR rate no more than $40 \%$ even for combined ICI therapy (36). Consequently, it is critical to identify the dominant population and prognostic indicators of ICIs. Some recommended predictive indexes for efficacy include PD-L1 expression, tumor mutation burden (TMB), microsatellite instability-high (MSI-H), and gut microbiome $(25,37,38)$. However, the biomarkers for irAEs are still blank, and the
A

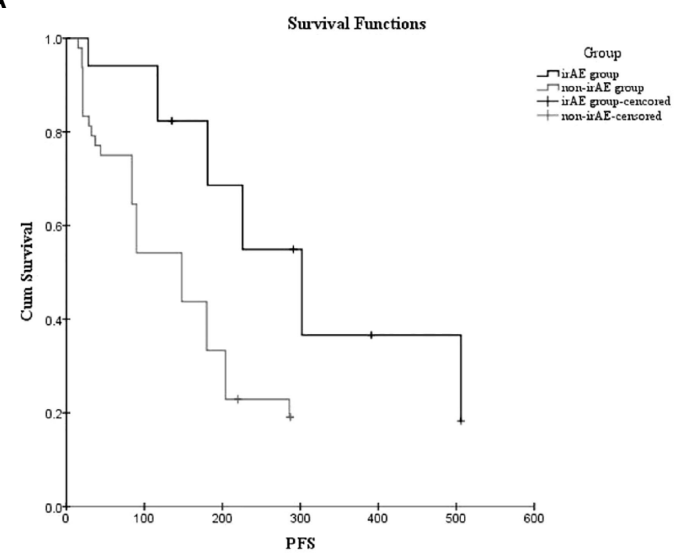

B

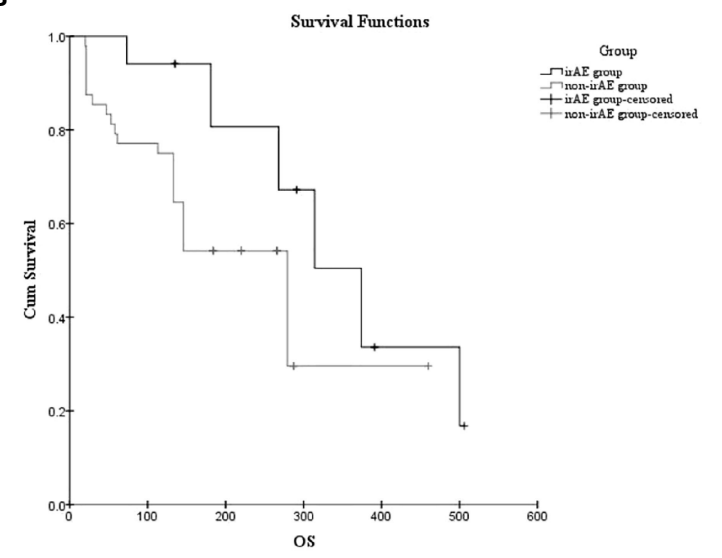

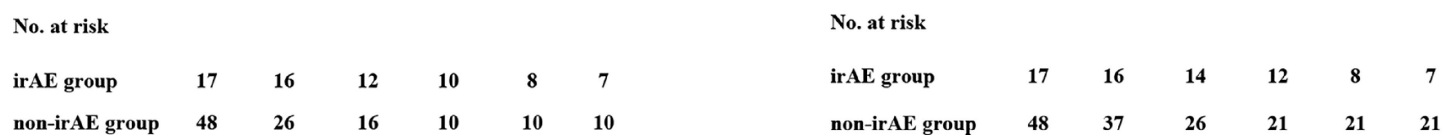

FIGURE 1 | The association of irAEs on the prognosis of HCC patients. (A) The Kaplan-Meier curve of progression-free survival (PFS). (B) The Kaplan-Meier curve of overall survival (OS). 
TABLE 3 | Kaplan-Meier survival curve of progression-free survival (PFS) and overall survival (OS).

\begin{tabular}{|c|c|c|c|c|}
\hline & Median PFS (days) & $p$-value & Median OS (days) & $p$-value \\
\hline \multicolumn{5}{|c|}{ irAE group vs. non-irAE group } \\
\hline irAE group & 302 (95\% Cl: 201 to 402) & 0.004 & 374 (95\% Cl: 291 to 456$)$ & 0.038 \\
\hline Non-irAE group & 148 (95\% Cl: 104 to 191$)$ & & 279 (95\% Cl: 205 to 352) & \\
\hline \multicolumn{5}{|c|}{ irAE-A group vs. non-irAE group } \\
\hline irAE-A group & 302 (95\% Cl: 206 to 398) & 0.002 & 374 (95\% Cl: 295 to 453$)$ & 0.034 \\
\hline Non-irAE group & 148 (95\% Cl: 104 to 191$)$ & & 279 (95\% Cl: 205 to 352) & \\
\hline \multicolumn{5}{|c|}{ irAE-B group vs. non-irAE group } \\
\hline irAE-B group & 181 (95\% Cl: 81 to 281$)$ & 0.642 & 314 (95\% Cl: 114 to 514$)$ & 0.613 \\
\hline Non-irAE group & 148 (95\% Cl: 104 to 191$)$ & & 279 (95\% Cl: 205 to 352) & \\
\hline \multicolumn{5}{|c|}{ irAE-A group vs. irAE-B group } \\
\hline irAE-A group & 302 (95\% Cl: 206 to 398) & 0.126 & 374 (95\% Cl: 295 to 453$)$ & 0.198 \\
\hline irAE-B group & 181 (95\% Cl: 81 to 281$)$ & & 314 (95\% Cl: 114 to 514$)$ & \\
\hline
\end{tabular}

PFS, progression-free survival; OS, overall survival; irAE-A group, the group with endocrine, dermatological, or gastrointestinal irAEs; irAE-B group, the group with other irAEs.

TABLE 4 | Univariate and multivariate analyses of OS with Cox regression models.

\begin{tabular}{|c|c|c|c|c|c|c|c|c|}
\hline \multirow[t]{2}{*}{ Covariate } & \multicolumn{4}{|c|}{ Univariate analysis $(n=65)$} & \multicolumn{4}{|c|}{ Multivariate analysis $(n=65)$} \\
\hline & HR & \multicolumn{2}{|c|}{$95.0 \% \mathrm{Cl}$} & $p$-value & HR & \multicolumn{2}{|c|}{$95.0 \% \mathrm{Cl}$} & $p$-value \\
\hline \multicolumn{9}{|l|}{ Group } \\
\hline irAE & Reference & & & & Reference & & & \\
\hline Non-irAE & 2.295 & 0.996 & 5.288 & 0.051 & 6.410 & 1.404 & 29.275 & 0.017 \\
\hline \multicolumn{9}{|l|}{ Gender } \\
\hline Female & Reference & & & & Reference & & & \\
\hline Male & 1.044 & 0.510 & 2.137 & 0.906 & 2.004 & 0.449 & 8.934 & 0.362 \\
\hline \multicolumn{9}{|l|}{ ECOG PS } \\
\hline$\leq 1$ & Reference & & & & Reference & & & \\
\hline$>1$ & 4.366 & 1.533 & 12.433 & 0.006 & 6.896 & 1.530 & 31.077 & 0.012 \\
\hline \multicolumn{9}{|c|}{ Treatment line } \\
\hline$\leq 2$ & Reference & & & & Reference & & & \\
\hline$\geq 3$ & 4.526 & 1.698 & 12.066 & 0.003 & 2.638 & 0.453 & 15.886 & 0.277 \\
\hline \multicolumn{9}{|l|}{ AFP } \\
\hline$<400$ & Reference & & & & Reference & & & \\
\hline$\geq 400$ & 2.765 & 1.206 & 6.342 & 0.016 & 1.540 & 0.476 & 4.987 & 0.471 \\
\hline \multicolumn{9}{|c|}{ Tumor number } \\
\hline$<3$ & Reference & & & & Reference & & & \\
\hline$\geq 3$ & 1.045 & 0.520 & 2.100 & 0.902 & 1.651 & 0.600 & 4.544 & 0.332 \\
\hline \multicolumn{9}{|l|}{ TNM } \\
\hline III & Reference & & & & Reference & & & \\
\hline IV & 5.996 & 2.722 & 13.211 & 0.001 & 5.427 & 2.323 & 12.679 & 0.001 \\
\hline \multicolumn{9}{|l|}{ Metastasis } \\
\hline No & Reference & & & & Reference & & & \\
\hline Yes & 2.308 & 1.159 & 4.597 & 0.017 & 3.265 & 1.141 & 9.348 & 0.027 \\
\hline
\end{tabular}

ECOG PS, Eastern Cooperative Oncology Group performance status; AFP, alpha fetoprotein.

occurrence of irAEs is usually detected only by regular detection of relevant indicators and clinical observation.

\section{LIMITATIONS}

This study has some limitations. First, the study is retrospective and conducted only in one center; the data from a multicenter should be evaluated in the further study. Second, the sample size was small; it may lead to large confidence intervals and affect the imprecision of the results. The larger sample size was valuable for stratified analysis to identify different types of irAEs responsible for ICI efficiency. Third, the effect of combined medicine on adverse reactions and prognosis in patients receiving combined medicine was not excluded in this study, although the combined medicine displayed a spectrum of different adverse events compared with ICIs for HCC treatment. The most common side effects for the systemic treatment of sorafenib and lenvatinib were hypertension, fatigue, proteinuria, and nausea, whereas the most common irAE involved the skin, followed by the gastrointestinal tract and endocrine system (39). Fourth, our study only included HCC patients with no preexisting autoimmune disease; the correlation between irAEs and prognosis in patients with preexisting autoimmune disease needs further exploration. Most immune-related side effects are managed without permanent drug discontinuation for ICItreated patients complicated with preexisting autoimmune disease, but patients with myasthenia gravis, multiple sclerosis, 
and inflammatory bowel disease (IBD) might have a higher risk of relapse and exacerbation $(16,40)$. However, to the best of our knowledge, this is the first work to reveal an association between irAEs and the efficacy of ICIs in HCC. Our goal is to identify potential predictive value for irAEs on treatment efficiency of ICIs from this pilot project first and to build the foundation for future research with a larger population. Findings from this study may also inspire other scientists to look for predictive values of irAEs in other tumors with ICI treatment.

\section{CONCLUSIONS}

Occurrence of irAEs was significantly associated with better clinical prognosis of HCC patients treated with immune checkpoint inhibitors; irAEs, particularly low-grade irAEs, were a predictable maker for better ICI treatment efficiency in HCC patients.

\section{DATA AVAILABILITY STATEMENT}

The original contributions presented in the study are included in the article/Supplementary Material. Further inquiries can be directed to the corresponding authors.

\section{REFERENCES}

1. Roth GA, Abate D, Abate KH, Abay SM, Abbafati C, Abbasi N, et al. Global, Regional, and National Age-Sex-Specific Mortality for 282 Causes of Death in 195 Countries and Territories, 1980-2017: A Systematic Analysis for the Global Burden of Disease Study 2017. Lancet (2018) 392(10159):1736-88. doi: $10.1016 / \mathrm{s} 0140-6736(18) 32203-7$

2. Sung H, Ferlay J, Siegel RL, Laversanne M, Soerjomataram I, Jemal A, et al. Global Cancer Statistics 2020: GLOBOCAN Estimates of Incidence and Mortality Worldwide for 36 Cancers in 185 Countries. CA Cancer J Clin (2021) 71(3):209-49. doi: 10.3322/caac.21660

3. Cheng A-L, Kang Y-K, Chen Z, Tsao C-J, Qin S, Kim JS, et al. Efficacy and Safety of Sorafenib in Patients in the Asia-Pacific Region With Advanced Hepatocellular Carcinoma: A Phase III Randomised, Double-Blind, PlaceboControlled Trial. Lancet Oncol (2009) 10(1):25-34. doi: 10.1016/s1470-2045 (08)70285-7

4. Kudo M, Finn RS, Qin S, Han K-H, Ikeda K, Piscaglia F, et al. Lenvatinib Versus Sorafenib in First-Line Treatment of Patients With Unresectable Hepatocellular Carcinoma: A Randomised Phase 3 Non-Inferiority Trial. Lancet (2018) 391(10126):1163-73. doi: 10.1016/s0140-6736(18)30207-1

5. Llovet JM, Ricci S, Mazzaferro V, Hilgard P, Gane E, Blanc J-F, et al. Sorafenib in Advanced Hepatocellular Carcinoma. N Engl J Med (2008) 359(4):378-90. doi: 10.1056/NEJMoa0708857

6. Abou-Alfa GK, Meyer T, Cheng AL, El-Khoueiry AB, Rimassa L, Ryoo BY, et al. Cabozantinib in Patients With Advanced and Progressing Hepatocellular Carcinoma. N Engl J Med (2018) 379(1):54-63. doi: 10.1056/ NEJMoa1717002

7. Bruix J, Qin S, Merle P, Granito A, Huang Y-H, Bodoky G, et al. Regorafenib for Patients With Hepatocellular Carcinoma Who Progressed on Sorafenib Treatment (RESORCE): A Randomised, Double-Blind, Placebo-Controlled, Phase 3 Trial. Lancet (2017) 389(10064):56-66. doi: 10.1016/s0140-6736(16) 32453-9

\section{ETHICS STATEMENT}

Written informed consent was obtained from the individual(s) for the publication of any potentially identifiable images or data included in this article.

\section{AUTHOR CONTRIBUTIONS}

All authors listed have made a substantial, direct, and intellectual contribution to the work and approved it for publication.

\section{FUNDING}

This work was supported by the Natural Science Foundation of China of Hebei Province (Grant No. H2019206428) and the Foundation of Hebei Provincial Department of Science and Technology \& Hebei Medical University (Grant No. 2020TXZH03).

\section{SUPPLEMENTARY MATERIAL}

The Supplementary Material for this article can be found online at: https://www.frontiersin.org/articles/10.3389/fimmu.2021. 794099/full\#supplementary-material

8. Zhu AX, Kang Y-K, Yen C-J, Finn RS, Galle PR, Llovet JM, et al. Ramucirumab After Sorafenib in Patients With Advanced Hepatocellular Carcinoma and Increased $\alpha$-Fetoprotein Concentrations (REACH-2): A Randomised, Double-Blind, Placebo-Controlled, Phase 3 Trial. Lancet Oncol (2019) 20(2):282-96. doi: 10.1016/s1470-2045(18)30937-9

9. Postow MA, Sidlow R, Hellmann MD. Immune-Related Adverse Events Associated With Immune Checkpoint Blockade. N Engl J Med (2018) 378 (2):158-68. doi: 10.1056/NEJMra1703481

10. Borghaei H, Paz-Ares L, Horn L, Spigel DR, Steins M, Ready NE, et al. Nivolumab Versus Docetaxel in Advanced Nonsquamous Non-Small-Cell Lung Cancer. N Engl J Med (2015) 373(17):1627-39. doi: 10.1056/ NEJMoa1507643

11. Weber JS, D'Angelo SP, Minor D, Hodi FS, Gutzmer R, Neyns B, et al. Nivolumab Versus Chemotherapy in Patients With Advanced Melanoma Who Progressed After Anti-CTLA-4 Treatment (CheckMate 037): A Randomised, Controlled, Open-Label, Phase 3 Trial. Lancet Oncol (2015) 16 (4):375-84. doi: 10.1016/s1470-2045(15)70076-8

12. Motzer RJ, Escudier B, McDermott DF, George S, Hammers HJ, Srinivas S, et al. Nivolumab Versus Everolimus in Advanced Renal-Cell Carcinoma. N Engl J Med (2015) 373(19):1803-13. doi: 10.1056/NEJMoa1510665

13. Ferris RL, Blumenschein G Jr, Fayette J, Guigay J, Colevas AD, Licitra L, et al. Nivolumab for Recurrent Squamous-Cell Carcinoma of the Head and Neck. N Engl J Med (2016) 375(19):1856-67. doi: 10.1056/NEJMoa1602252

14. El-Khoueiry AB, Sangro B, Yau T, Crocenzi TS, Kudo M, Hsu C, et al. Nivolumab in Patients With Advanced Hepatocellular Carcinoma (CheckMate 040): An Open-Label, Non-Comparative, Phase 1/2 Dose Escalation and Expansion Trial. Lancet (2017) 389(10088):2492-502. doi: 10.1016/s0140-6736(17)31046-2

15. Zhu AX, Finn RS, Edeline J, Cattan S, Ogasawara S, Palmer D, et al. Pembrolizumab in Patients With Advanced Hepatocellular Carcinoma Previously Treated With Sorafenib (KEYNOTE-224): A Non-Randomised, Open-Label Phase 2 Trial. Lancet Oncol (2018) 19(7):940-52. doi: 10.1016/ s1470-2045(18)30351-6 
16. Abd El Aziz MA, Facciorusso A, Nayfeh T, Saadi S, Elnaggar M, Cotsoglou C, et al. Immune Checkpoint Inhibitors for Unresectable Hepatocellular Carcinoma. Vaccines (2020) 8(4):616. doi: 10.3390/vaccines8040616

17. Yau T, Park JW, Finn RS, Cheng AL, Mathurin P, Edeline J, et al. LBA38_PR CheckMate 459: A Randomized, Multi-Center Phase III Study of Nivolumab (NIVO) vs Sorafenib (SOR) as First-Line (1L) Treatment in Patients (Pts) With Advanced Hepatocellular Carcinoma (aHCC). Ann Oncol (2019) 30: v874-5. doi: 10.1093/annonc/mdz394.029

18. Finn R, Ryoo B, Merle P, Kudo M, Bouattour M, Lim H, et al. Pembrolizumab As Second-Line Therapy in Patients With Advanced Hepatocellular Carcinoma in KEYNOTE-240: A Randomized, Double-Blind, Phase III Trial. J Clin Oncol (2020) 38(3):193-202. doi: 10.1200/jco.19.01307

19. Qin S, Ren Z, Feng YH, Yau T, Wang B, Zhao H, et al. Atezolizumab Plus Bevacizumab Versus Sorafenib in the Chinese Subpopulation With Unresectable Hepatocellular Carcinoma: Phase 3 Randomized, Open-Label IMbrave150 Study. Liver Cancer (2021) 10(4):296-308. doi: 10.1159/ 000513486

20. Galle PR, Finn RS, Qin S, Ikeda M, Zhu AX, Kim T-Y, et al. Patient-Reported Outcomes With Atezolizumab Plus Bevacizumab Versus Sorafenib in Patients With Unresectable Hepatocellular Carcinoma (IMbrave150): An Open-Label, Randomised, Phase 3 Trial. Lancet Oncol (2021) 22(7):991-1001. doi: 10.1016/S1470-2045(21)00151-0

21. Rizzo A, Ricci AD, Brandi G. Atezolizumab in Advanced Hepatocellular Carcinoma: Good Things Come to Those Who Wait. Immunotherapy (2021) 13(8):637-44. doi: 10.2217/imt-2021-0026

22. Rizzo A, Dadduzio V, Ricci AD, Massari F, Di Federico A, Gadaleta-Caldarola G, et al. Lenvatinib Plus Pembrolizumab: The Next Frontier for the Treatment of Hepatocellular Carcinoma? Expert Opin Invest Drugs (2021), 1-8. doi: 10.1080/13543784.2021.1948532

23. Finn RS, Ikeda M, Zhu AX, Sung MW, Baron AD, Kudo M, et al. Phase Ib Study of Lenvatinib Plus Pembrolizumab in Patients With Unresectable Hepatocellular Carcinoma. J Clin Oncol (2020) 38(26):2960-70. doi: $10.1200 /$ jco.20.00808

24. Rizzo A, Brandi G. Biochemical Predictors of Response to Immune Checkpoint Inhibitors in Unresectable Hepatocellular Carcinoma. Cancer Treat Res Commun (2021) 27:100328. doi: 10.1016/j.ctarc.2021.100328

25. Zheng Y, Wang T, Tu X, Huang Y, Zhang H, Tan D, et al. Gut Microbiome Affects the Response to Anti-PD-1 Immunotherapy in Patients With Hepatocellular Carcinoma. J Immunother Cancer (2019) 7(1):193. doi: 10.1186/s40425-019-0650-9

26. Voskens CJ, Goldinger SM, Loquai C, Robert C, Kaehler KC, Berking C, et al. The Price of Tumor Control: An Analysis of Rare Side Effects of Anti-CTLA-4 Therapy in Metastatic Melanoma From the Ipilimumab Network. PloS One (2013) 8(1):e53745. doi: 10.1371/journal.pone.0053745

27. Teraoka S, Fujimoto D, Morimoto T, Kawachi H, Ito M, Sato Y, et al. Early Immune-Related Adverse Events and Association With Outcome in Advanced Non-Small Cell Lung Cancer Patients Treated With Nivolumab: A Prospective Cohort Study. J Thorac Oncol (2017) 12(12):1798-805. doi: 10.1016/j.jtho.2017.08.022

28. Robert C, Long GV, Brady B, Dutriaux C, Maio M, Mortier L, et al. Nivolumab in Previously Untreated Melanoma Withoutbrafmutation. $N$ Engl $J$ Med (2015) 372(4):320-30. doi: 10.1056/NEJMoa1412082

29. Weber JS, Hodi FS, Wolchok JD, Topalian SL, Schadendorf D, Larkin J, et al. Safety Profile of Nivolumab Monotherapy: A Pooled Analysis of Patients With Advanced Melanoma. J Clin Oncol (2017) 35(7):785-92. doi: 10.1200/ JCO.2015.66.1389

30. Brahmer JR, Lacchetti C, Schneider BJ, Atkins MB, Brassil KJ, Caterino JM, et al. Management of Immune-Related Adverse Events in Patients Treated With Immune Checkpoint Inhibitor Therapy: American Society of Clinical Oncology Clinical Practice Guideline. J Clin Oncol (2018) 36(17):1714-68. doi: 10.1200/jco.2017.77.6385
31. Toi Y, Sugawara S, Kawashima Y, Aiba T, Kawana S, Saito R, et al. Association of Immune-Related Adverse Events With Clinical Benefit in Patients With Advanced Non-Small-Cell Lung Cancer Treated With Nivolumab. Oncologist (2018) 23(11):1358-65. doi: 10.1634/theoncologist.2017-0384

32. Matsuoka H, Hayashi T, Takigami K, Imaizumi K, Shiroki R, Ohmiya N, et al. Correlation Between Immune-Related Adverse Events and Prognosis in Patients With Various Cancers Treated With Anti PD-1 Antibody. BMC Cancer (2020) 20(1):656. doi: 10.1186/s12885-020-07142-3

33. Nakamura $Y$, Tanaka R, Asami $Y$, Teramoto $Y$, Imamura $T$, Sato $S$, et al. Correlation Between Vitiligo Occurrence and Clinical Benefit in Advanced Melanoma Patients Treated With Nivolumab: A Multi-Institutional Retrospective Study. J Dermatol (2017) 44(2):117-22. doi: 10.1111/13468138.13520

34. Ricciuti B, Genova C, De Giglio A, Bassanelli M, Dal Bello MG, Metro G, et al. Impact of Immune-Related Adverse Events on Survival in Patients With Advanced Non-Small Cell Lung Cancer Treated With Nivolumab: LongTerm Outcomes From a Multi-Institutional Analysis. J Cancer Res Clin Oncol (2019) 145(2):479-85. doi: 10.1007/s00432-018-2805-3

35. Zhou X, Yao Z, Yang H, Liang N, Zhang X, Zhang F. Are Immune-Related Adverse Events Associated With the Efficacy of Immune Checkpoint Inhibitors in Patients With Cancer? A Systematic Review and MetaAnalysis. BMC Med (2020) 18(1):87. doi: 10.1186/s12916-020-01549-2

36. Ito A, Kondo S, Tada K, Kitano S. Clinical Development of Immune Checkpoint Inhibitors. BioMed Res Int (2015) 2015:605478. doi: 10.1155/ 2015/605478

37. Xu Y, Wan B, Chen X, Zhan P, Zhao Y, Zhang T, et al. The Association of PDL1 Expression With the Efficacy of Anti-PD-1/PD-L1 Immunotherapy and Survival of Non-Small Cell Lung Cancer Patients: A Meta-Analysis of Randomized Controlled Trials. Transl Lung Cancer Res (2019) 8(4):413-28. doi: $10.21037 /$ tlcr.2019.08.09

38. Cao D, Xu H, Xu X, Guo T, Ge W. High Tumor Mutation Burden Predicts Better Efficacy of Immunotherapy: A Pooled Analysis of 103078 Cancer Patients. Oncoimmunology (2019) 8(9):e1629258. doi: 10.1080/2162402X. 2019.1629258

39. Wang PF, Chen Y, Song SY, Wang TJ, Ji WJ, Li SW, et al. Immune-Related Adverse Events Associated With Anti-PD-1/PD-L1 Treatment for Malignancies: A Meta-Analysis. Front Pharmacol (2017) 8:730. doi: $10.3389 /$ fphar.2017.00730

40. Meserve J, Facciorusso A, Holmer AK, Annese V, Sandborn WJ, Singh S. Systematic Review With Meta-Analysis: Safety and Tolerability of Immune Checkpoint Inhibitors in Patients With Pre-Existing Inflammatory Bowel Diseases. Aliment Pharmacol Ther (2021) 53(3):374-82. doi: 10.1111/ apt.16217

Conflict of Interest: The authors declare that the research was conducted in the absence of any commercial or financial relationships that could be construed as a potential conflict of interest.

Publisher's Note: All claims expressed in this article are solely those of the authors and do not necessarily represent those of their affiliated organizations, or those of the publisher, the editors and the reviewers. Any product that may be evaluated in this article, or claim that may be made by its manufacturer, is not guaranteed or endorsed by the publisher.

Copyright $\odot 2021 \mathrm{Xu}$, Lai, Zhao, Zhao, Zhao and Guo. This is an open-access article distributed under the terms of the Creative Commons Attribution License (CC BY). The use, distribution or reproduction in other forums is permitted, provided the original author(s) and the copyright owner(s) are credited and that the original publication in this journal is cited, in accordance with accepted academic practice. No use, distribution or reproduction is permitted which does not comply with these terms. 